\title{
Disjointly Strictly Singular Inclusions of Symmetric Spaces
}

\author{
S.V.Astashkin \\ Samara State University, 443011 Samara 11, Russia
}

\begin{abstract}
In this paper, the disjoint strict singularity of inclusions of symmetric spaces of functions on an interval is considered. A condition for the presence of a "gap" between spaces sufficient for the inclusion of one of these spaces into the other to be disjointly strictly singular is found. The condition is stated in terms of fundamental functions of spaces and is exact in a certain sense. In parallel, necessary and sufficient conditions for an inclusion of Lorentz spaces to be disjointly strictly singular (and similar conditions for Marcinkiewicz spaces) are obtained and certain other assertions are proved.
\end{abstract}

Keywords: Banach space, disjointly strictly singular operator, inclusion operator, symmetric space, fundamental function, Lorentz space, Marcinkiewicz space, Orlicz space

\section{Introduction}

Recall that a bounded linear operator $T$ from a Banach space $X$ into a Banach space $Y$ is called strictly singular (or a Cato operator) if $X$ does not contain an infinitedimensional subspace $Z$ such that the restriction of $T$ to $Z$ is an isomorphism.

In recent decades the class of strictly singular operators has been extensively studied (see the references in, e.g., the monograph [1]). One of the historically first results important for our purposes is the Grothendick theorem on strict singularity of the identity inclusion operator from $L_{\infty}(\Omega, \mu)$ into $L_{p}(\Omega, \mu)$, where $1 \leq p<\infty$ and $\mu$ is a probability measure on $\Omega$ (see [2] or [3, Theorem 5.2]). However, as a rule, the identity inclusion operator from one symmetric space into another (the definition is given below) is not strictly singular because of the existence of "through" subspaces (such as the subspace generated by the Rademacher functions [4]). In part because of this, the close notion of disjointly strictly singular operator was introduced in 1989 $[5]$.

A bounded linear operator $T$ from a Banach lattice $X$ into a Banach space $Y$ is called disjointly strictly singular (or has the DSS property) if there exists no sequence of nonzero disjoint vectors $\left\{x_{n}\right\}_{n=1}^{\infty}$ in $X$ such that the restriction of $T$ to their closed linear hull $\left[x_{n}\right]$ is an isomorphism.

Clearly, any strictly singular operator is a DSS operator. A simple example shows that the converse is not true. For instanse, the identity inclusion operator $I: L_{p}[0,1] \rightarrow$ $L_{q}[0,1](1 \leq q<p \leq \infty)$ has the DSS property, because the closed linear hull in $L_{r}$ of disjoint functions $x_{n} \in L_{r}[0,1]$ is isomorphic to $l_{r}(1 \leq r \leq \infty):\left[x_{n}\right]_{r} \approx l_{r}$. However, if $p<\infty$, then Khintchine's inequality [6] implies that $\left[r_{n}\right]_{p} \approx\left[r_{n}\right]_{q} \approx l_{2}\left(r_{n}\right.$ are Rademacher functions), and therefore $I$ is not strictly singular. At the same time, 
it is easy to show that if $X$ has a Schauder basis of disjoint vectors, then the class of DSS operators on $X$ coincides with the class of strictly singular operators [7].

The notion of DSS operator proved important in studies of the geometric properties of function spaces. For example, the existence of operators without the DSS property makes it possible to construct complemented subspaces that admit "nonstandard" projections onto them $[5,7]$.

The goal of this paper is to study the following question: when does the identity inclusion operator (throughout, we denote it by $I$ ) from one symmetric space in another have the DSS property? The conditions are stated in terms of fundamental functions of these spaces.

If $z=z(t)$ is measurable on $[0,1]$ with respect to the Lebesgue measure $\mu$, then we call the function $n_{z}(\tau)=\mu\{t:|z(t)|>\tau\}(\tau>0)$ the distribution function of $z$. Two functions $x(t)$ and $y(t)$ are called equimeasurable if $n_{x}(\tau)=n_{y}(\tau)$ for $\tau>0$.

Recall that a Banach space $E$ of measurable functions on $[0,1]$ is called a symmetric space (briefly is an SS) if the following conditions hold:

(1) if $y \in E$ and $|x(t)| \leq|y(t)|$, then $x \in E$ and $\|x\| \leq\|y\|$;

(2) if $y \in E$ and functions $x(t)$ and $y(t)$ are equimeasurable, then $x \in E$ and $\|x\|=\|y\|$.

The fundamental function of an SS $E$ is defined by $f_{E}(t)=\left\|\chi_{(0, t)}\right\|_{E}$, where, as usual, $\chi_{U}(t)=1(t \in U), \chi_{U}(t)=0(t \notin U)$. The function $f_{E}(t)$ is quasiconcave on $(0,1]$ [8, p.137], i.e., it is nonnegative, increasing, and $f_{E}(t) / t$ decreases. As is known (see, e.g., [8, p.70]), such a function is equivalent to its least concave majorant. Throughout, $G$ denotes the class of all positive increasing functions concave on $(0,1]$.

An important example of an SS is an Orlicz space. Let $N(t)$ be an increasing convex function on $[0, \infty)$ such that $N(0)=0$ and $N(\infty)=\infty$. The Orlicz space $L_{N}$ consists of all functions $x=x(t)$ measurable on $[0,1]$ and such that

$$
\int_{T} N\left(\frac{|x(t)|}{u}\right) d \mu<\infty
$$

for some $u>0$; the norm of this space is

$$
\|x\|=\inf \left\{u>0: \int_{T} N\left(\frac{|x(t)|}{u}\right) d \mu \leq 1\right\} .
$$

Direct calculation shows that the fundamental function of the space $L_{N}$ is $f_{N}(t)=$ $1 / N^{-1}(1 / t)\left(N^{-1}(u)\right.$ is the inverse of $\left.N(u)\right)[9]$.

In [5], the following disjoint strict singularity theorem for inclusions of $L_{N}$ into $L_{M}$ is proved.

Theorem. If $L_{N} \subset L_{M}$, then the following conditions are equivalent:

(1) the inclusion $I: L_{N} \rightarrow L_{M}$ is a DSS operator;

(2) for any $n=1,2,$. and $\mathcal{K}>0$, there exist $1 \leq x_{1}<x_{2}<\ldots<x_{n}$ and $c_{1}>0, . ., c_{n}>0$ such that

$$
\sum_{i=1}^{n} c_{i} N\left(t x_{i}\right) \geq \mathcal{K} \sum_{i=1}^{n} c_{i} M\left(t x_{i}\right) \quad \text { for } t \geq 1 .
$$


Let us show that condition (2) follows from the relation

$$
\lim _{t \rightarrow+0} \frac{f_{M}(t)}{f_{N}(t)}=0
$$

where $f_{N}$ and $f_{M}$ are the fundamental functions of the respective Orlicz spaces. Indeed, this relation implies that $N^{-1}(t) \leq h M^{-1}(t)$ for an arbitrary positive $h \leq 1$ and $t \geq t_{0}$ and, since $N(t)$ is convex, $M(t) \leq N(h t) \leq h N(t)$, if $t \geq M^{-1}\left(t_{0}\right)$. Therefore,

$$
\lim _{t \rightarrow \infty} \frac{M(t)}{N(t)}=0
$$

and condition (2) holds.

Quite naturally, this observation leads us to the following general problem.

Suppose that functions $\varphi \in G$ and $\psi \in G$ satisfy the condition

(A) $\lim _{t \rightarrow+0} \psi(t) / \varphi(t)=0$

and are, respectively, the fundamental functions of symmetric spaces $E$ and $F$ such that $E \subset F$. Does the identity inclusion operator $I: E \rightarrow F$ have the DSS property?

In what follows, we show that the answer to this question for "classical" symmetric spaces (such as the Lorentz and Marcinkiewicz spaces) as well as for Orlicz spaces is positive. Moreover, it is so for an inclusion of a Lorentz space into an arbitrary SS (and, vice versa, of an arbitrary SS into a Marcinkiewicz space).

However, in the general case, this is not true: this paper contains an example of two symmetric spaces $E$ and $F$ such that $E \subset F$ and their fundamental functions satisfy condition (A), but $I: E \rightarrow F$ is not a DSS operator.

At the same time, it is possible to state a condition on fundamental functions stronger than (A) under which the answer to the stated question is positive for all symmetric spaces. First, recall the definition of the dilation function.

For a positive function $f$ on $(0,1]$, its dilation function $\mathcal{M}_{f}(t)$ is defined as

$$
\mathcal{M}_{f}(t)=\sup \left\{\frac{f(s t)}{f(s)}: 0<s \leq \min \left(1, \frac{1}{t}\right)\right\} \quad(t>0) .
$$

Since $\mathcal{M}_{f}(t)$ is semimultiplicative, there exist numbers

$$
\gamma_{f}=\lim _{t \rightarrow 0} \frac{\ln \mathcal{M}_{f}(t)}{\ln t} \text { and } \quad \delta_{f}=\lim _{t \rightarrow \infty} \frac{\ln \mathcal{M}_{f}(t)}{\ln t}
$$

which are called, respectively, the lower and upper dilation indices of function $f$. If $\varphi \in G$, then we have $0 \leq \gamma_{\varphi} \leq \delta_{\varphi} \leq 1[8$, p.76]

Let us introduce one more condition on the functions $\varphi$ and $\psi$ from the class $G$ :

(B) $\gamma_{\psi / \varphi}>0$

The definition of lower dilation index readily implies that condition (A) follows from (B). The converse, of course, is not true: it suffices to take for $\varphi$ and $\psi$ functions differing by a logarithmic factor (see also the proof of Theorem 3 ).

We shall show that, if (B) holds, then operator $I: E \rightarrow F$ will be a DSS operator for arbitrary symmetric spaces $E$ and $F, E \subset F$, with fundamental functions $\varphi$ and $\psi$, respectively. This result generalizes and simultaneously refines a similar theorem for the Orlicz spaces proved in [7]. In parallel, we shall show that condition (A) is necessary and sufficient for the identity inclusion operator from one Lorentz space into another to have the DSS property (and similar assertion for Marcinkiewicz spaces). These results also supplement the theorem for Orlicz spaces proved in [5] and cited above. 


\section{$\S 1$. The inclusions $\Lambda(\varphi) \subset F$ and $E \subset M(\theta)$}

For $\varphi \in G$, the Lorentz space $\Lambda(\varphi)$ consists of all functions $x=x(s)$ measurable on $[0,1]$ and such that

$$
\|x\|_{\Lambda(\varphi)}=\int_{0}^{1} x^{*}(s) d \varphi(s)<\infty,
$$

where $x^{*}(s)$ is a decreasing left-continuous rearrangement of the function $|x(s)|[8$, p.83]. Clearly, the fundamental function of the Lorentz space $\Lambda(\varphi)$ is $f_{\Lambda(\varphi)}(t)=\varphi(t)$.

Theorem 1. Suppose that the functions $\varphi \in G$ and $\psi \in G$ satisfy $(A)$ and $F$ is an $S S$ on $[0,1]$ with fundamental function $\psi(t)$. Then $\Lambda(\varphi) \subset F$ and the identity inclusion $I: \Lambda(\varphi) \rightarrow F$ is a DSS operator.

Proof. Condition (A) and the continuity of the concave functions $\varphi$ and $\psi$ at $t>0$ imply that $\psi(t) \leq C_{1} \varphi(t)$ for $t \in[0,1]$. By the definition of the norm of a Lorentz space, we have $\Lambda(\varphi) \subset \Lambda(\psi)$. The Lorentz space with a given fundamental function is minimal among the symmetric spaces with the same fundamental function [8, p.160]; therefore, $\Lambda(\varphi) \subset F$.

Suppose that $I: \Lambda(\varphi) \rightarrow F$ has not the DSS property. Then there exists a sequence of nonzero disjoint functions $x_{n} \geq 0$ such that

$$
\left\|x_{n}\right\|_{\Lambda(\varphi)} \leq C_{2}\left\|x_{n}\right\|_{F} \text { for } n=1,2, . .
$$

By condition (A), for any $0<\varepsilon<1$, there exists an $h>0$ such that

$$
\psi(t) \leq \varepsilon \varphi(t)
$$

for all positive $t<h$. Choose $N$ so that, for $n \geq N, \mu\left(g_{n}\right)<h$, where $g_{n}=\{t \in$ $\left.[0,1]: x_{n}(t) \neq 0\right\}$.

The subset of finite-valued functions is dense in any Lorentz space on $[0,1][8$, p.149]. Therefore, for each $n \geq N$, there exists a function

$$
y_{n}(t)=\sum_{k=1}^{m_{n}} a_{k}^{n} \chi_{e_{k}^{n}}, \quad \text { where } a_{k}^{n} \geq 0, e_{1}^{n} \supset e_{2}^{n} \supset \ldots \supset e_{m_{n}}^{n}, \quad \text { and } \quad \mu\left(e_{1}^{n}\right)<h,
$$

for which

$$
\max \left(\left\|x_{n}-y_{n}||_{\Lambda(\varphi)},\right\| x_{n}-y_{n} \|_{\Lambda(\psi)}\right)<\varepsilon \min \left(\left\|x_{n}\right\|_{\Lambda(\varphi)},\left\|x_{n}\right\|_{\Lambda(\psi)}\right) .
$$

Hence $\left\|x_{n}\right\|_{\Lambda(\psi)}-\left\|y_{n}\right\|_{\Lambda(\psi)} \leq \varepsilon\left\|x_{n}\right\|_{\Lambda(\psi)}$, and [8, p.160] and (2) imply that

$$
\begin{aligned}
&\left\|x_{n}\right\|_{F} \leq\left\|x_{n}\right\|_{\Lambda(\psi)} \leq 1 /(1-\varepsilon) \| y_{n} \|_{\Lambda(\psi)}=1 /(1-\varepsilon) \sum_{k=1}^{m_{n}} a_{k}^{n} \psi\left(\mu\left(e_{k}^{n}\right)\right) \leq \\
& \leq \varepsilon /(1-\varepsilon) \sum_{k=1}^{m_{n}} a_{k}^{n} \varphi\left(\mu\left(e_{k}^{n}\right)\right)=\varepsilon /(1-\varepsilon)\left\|y_{n}\right\|_{\Lambda(\varphi)} .
\end{aligned}
$$

In addition, by (3),

$$
\left\|y_{n}\right\|_{\Lambda(\varphi)} \leq(1+\varepsilon)\left\|x_{n}\right\|_{\Lambda(\varphi)}
$$


Thus,

$$
\left\|x_{n}\right\|_{F} \leq \frac{\varepsilon(1+\varepsilon)}{1-\varepsilon}\left\|x_{n}\right\|_{\Lambda(\varphi)}
$$

This inequality with an $\varepsilon>0$ satisfying

$$
\frac{1-\varepsilon}{\varepsilon(1+\varepsilon)}>C_{2}
$$

contradicts (1).

Corollary 1. Suppose that $\varphi \in G, \psi \in G$, and $\psi(t) \leq C_{1} \varphi(t)$ for $t \in(0,1]$. The following conditions are equivalent:

(1) (A) holds;

(2) the inclusion $I: \Lambda(\varphi) \rightarrow \Lambda(\psi)$ is a DSS operator;

(3) there exist no sequence of nonzero disjoint functions $\left\{x_{n}\right\}$ and no constant $C_{2}>0$ such that

$$
\left\|x_{n}\right\|_{\Lambda(\varphi)} \leq C_{2}\left\|x_{n}\right\|_{\Lambda(\psi)} \text { for } n=1,2, \ldots
$$

Proof. The implications $(1) \rightarrow(2)$ and $(2) \rightarrow(3)$ follow from Theorem 1 and the definition of a DSS operator, respectively.

Suppose that condition (A) does not hold, i.e., that

$$
\limsup _{t \rightarrow 0} \frac{\psi(t)}{\varphi(t)}>0 \text {. }
$$

Then there exist a sequence $\left\{t_{k}\right\} \subset(0,1]$ and a constant $C_{2}>0$ such that we have $\sum_{n=1}^{\infty} t_{n} \leq 1$ and $\varphi\left(t_{n}\right) \leq C_{2} \psi\left(t_{n}\right)$ for $n=1,2, \ldots$ The functions $x_{n}=\chi_{e_{n}}$, where $e_{n} \subset[0,1]$ are disjoint and $\mu\left(e_{n}\right)=t_{n}$, satisfy (4). Therefore, (3) implies (1); this completes the proof of Corollary 1.

Let $\theta$ be a function from $G$. The Marcinkiewicz space $M(\theta)$ consists of all functions $x=x(s)$ measurable on $[0,1]$ and such that

$$
\|x\|_{M(\theta)}=\sup _{0<t \leq 1} \frac{1}{\theta(t)} \int_{0}^{t} x^{*}(s) d s<\infty .
$$

The fundamental function of the space $M(\theta)$ is $f_{M(\theta)}(t)=\tilde{\theta}(t)=t / \theta(t)$.

Theorem 2. Let the functions $\varphi \in G$ and $\psi \in G$ satisfy condition (A). If $E$ is an $S S$ on $[0,1]$ with fundamental function $\varphi(t)$, then $E \subset M(\tilde{\psi})$ and the identity inclusion $I: E \rightarrow M(\tilde{\psi})$ is a DSS operator.

Proof. Since any Marcinkiewicz space is maximal among all symmetric spaces with the same fundamental function [8, p.162], we have $E \subset M(\tilde{\psi})$.

By condition $(\mathrm{A}), \psi(t) \leq C_{1} \varphi(t)$ for $t \in(0,1]$; therefore, by the definition of a Marcinkiewicz space, $M(\tilde{\varphi}) \subset M_{\sim}(\tilde{\psi})$. Hence, $E \subset M(\tilde{\psi})$.

Suppose that $I: E \rightarrow M(\tilde{\psi})$ is not a DSS operator. Then, in particular, there exists a sequence of disjoint functions $x_{n}$ such that

$$
\left\|x_{n}\right\|_{M(\tilde{\psi})}=1 \text { and }\left\|x_{n}\right\|_{M(\tilde{\varphi})} \leq C_{2} \text { for } n=1,2, \ldots
$$


Choose a $t_{k} \in(0,1]$ for which

$$
\int_{0}^{t_{k}} x_{k}^{*}(s) d s \geq \frac{1}{2} \tilde{\psi}\left(t_{k}\right) \quad(k=1,2, . .) .
$$

Since the functions $x_{k}$ are disjoint, we can assume that $t_{k} \rightarrow 0$. Therefore,

$$
\left\|x_{k}\right\|_{M(\tilde{\varphi})} \geq \frac{1}{\tilde{\varphi}\left(t_{k}\right)} \int_{0}^{t_{k}} x_{k}^{*}(s) d s \geq \frac{\varphi\left(t_{k}\right)}{2 \psi\left(t_{k}\right)} .
$$

By $(\mathrm{A}),\left\|x_{k}\right\|_{M(\tilde{\varphi})} \rightarrow \infty$ as $k \rightarrow \infty$, which contradicts condition (5).

This completes the proof of Theorem 2.

Corollary 2. Suppose that $\varphi \in G, \psi \in G$, and $\psi(t) \leq C_{1} \varphi(t)$ for $t \in(0,1]$. The following conditions are equivalent:

(1) (A) holds;

(2) the inclusion $I: M(\tilde{\varphi}) \rightarrow M(\tilde{\psi})$ is a DSS operator;

(3) there exist no sequence of nonzero disjoint functions $x_{n}$ and no constant $C_{2}>0$ such that for some $C_{2}>0$

$$
\left\|x_{n}\right\|_{M(\tilde{\varphi})} \leq C_{2}\left\|x_{n}\right\|_{M(\tilde{\psi})} \text { for } n=1,2, \ldots
$$

The proof of Corollary 2 is similar to the proof of Corollary 1.

Corollary 3. For an arbitrary $S S E \neq L_{1}$ on $[0,1]$, the inclusion $I: E \rightarrow L_{1}$ is a DSS operator.

Proof. First, $L_{1}=M(1)$ and an arbitrary SS $E$ is embedded in $L_{1}$ [8, p.124]. If $f_{E}(t)=\varphi(t)$, then the function $t / \varphi(t)$ increases, because $\varphi$ is concave. Therefore, condition $(\mathrm{A})$ is violated if and only if $\varphi(t) \approx t$ (i.e., if and only if $C_{1} t \leq \varphi(t) \leq C_{2} t$ for some $C_{1}>0$ and $C_{2}>0$ ), and $E=L_{1}$. It remains to apply Theorem 2 .

Remark 1. The assertion of Corollary 3 was proved in [10] in a different way.

Remark 2. Arguing as in the proof of Corollary 3 and applying Theorem 1, we can readily show that the inclusion $I: L_{\infty} \rightarrow E$ is a DSS operator for any $\operatorname{SS} E \neq L_{\infty}$. Moreover, it is shown in [11] that this operator is even strictly singular. This generalizes the Grothendieck theorem mentioned in the introduction.

In the next section we show that, generally, condition (A) is not sufficient for the inclusion of an SS with fundamental function $\varphi$ into an SS with fundamental function $\psi$ to have the DSS property.

\section{$\S 2$. An example of symmetric spaces $E$ and $F$ such that $E \subset F$ and their fundamental functions satisfy condition (A), but $I: E \rightarrow F$ is not a DSS operator}

Theorem 3. There exist two symmetric spaces $E$ and $F$ on $[0,1]$ with fundamental functions $\varphi$ and $\psi$, respectively, such that $E \subset F, \varphi$ and $\psi$ satisfy condition (A), and the operator $I: E \rightarrow F$ has not the DSS property. 
Proof. Let the SS $E$ be the Marcinkiewicz space $M(\tilde{\psi})$ with $\tilde{\psi}(t)=t / \psi(t)$, where

$$
\psi(t)=t^{1 / 2} \log _{2}^{1 / 2} \frac{4}{t}, \quad 0<t \leq 1 .
$$

It is readily verified that $\psi$ is an increasing concave function on $[0,1]$ and $\gamma_{\psi}=\delta_{\psi}=$ $1 / 2$. Therefore, by $[8$, p.156],

$$
\|x\|_{M(\tilde{\psi})} \approx \sup _{0<t \leq 1}\left\{x^{*}(t) \psi(t)\right\} .
$$

Let us define the space $F$. We put $b_{k}=(k+2)^{-1 / 2} 2^{k / 2}$ and $z_{k}(t)=b_{k} \chi_{\left(0,2^{-k}\right]}(t)$ and define a sequence of numbers $n_{0}=1<n_{1}<n_{2}<\ldots<n_{m}<\ldots$ by setting

$$
n_{m+1}=\max \left\{n=1,2, . .: \sum_{k=n_{m}}^{n-1} \frac{1}{k+2} \leq 1\right\}
$$

and a sequence of functions $w_{m}=w_{m}(t)$ by setting

$$
w_{m}(t)=\max _{n_{m} \leq k<n_{m+1}} z_{k}(t), \quad m=0,1, . .
$$

Since $\left\{b_{k}\right\}$ increases, $(7)$ implies that the norms of $w_{m}$ in $L_{2}$ satisfy the inequalities:

$$
\left\|w_{m}\right\|_{2}^{2} \geq \sum_{k=n_{m}}^{n_{m+1}-1} b_{k}^{2} 2^{-k-1}=1 / 2 \sum_{k=n_{m}}^{n_{m+1}-1} \frac{1}{k+2} \geq \frac{1}{4}
$$

and

$$
\left\|w_{m}\right\|_{2}^{2} \leq \sum_{k=n_{m}}^{n_{m+1}-1} b_{k}^{2} 2^{-k}=\sum_{k=n_{m}}^{n_{m+1}-1} \frac{1}{k+2} \leq 1
$$

Therefore,

$$
\frac{1}{2} \leq\left\|w_{m}\right\|_{2} \leq 1
$$

Consider $\chi_{b}=b^{-1 / 2} \chi_{(0, b)}, \bar{w}_{m}=w_{m} /\left\|w_{m}\right\|_{2}$, and $V=\left\{\chi_{b}\right\}_{0<b \leq 1} \bigcup\left\{\bar{w}_{m}\right\}_{m=0}^{\infty}$. Let $F$ be the set of all functions $x=x(t)$ measurable on $[0,1]$ and satisfying

$$
\|x\|=\sup \left\{\int_{0}^{1} x^{*}(t) v(t) d t: v \in V\right\}<\infty .
$$

Then $F$ is an SS on $[0,1]$ as the intersection of Lorentz spaces determined by the functions $\int_{0}^{t} v(s) d s$ with $v \in V$. In addition, the definition of $F$ implies that $\|x\|_{M\left(t^{1 / 2}\right)} \leq$ $\|x\|_{F} \leq\|x\|_{2}$. Therefore the fundamental functions $f_{E}(t)=\psi(t)$ and $f_{F}(t)=t^{1 / 2}$ of the spaces $E$ and $F$ satisfy condition (A).

Let us prove that

$$
M(\tilde{\psi}) \subset F .
$$

By (6), it is suffices to show that $1 / \psi \in F$. Indeed,

$$
\int_{0}^{1} \chi_{b}(t) \frac{d t}{\psi(t)}=2 b^{-1 / 2} \int_{0}^{b} \frac{d\left(t^{1 / 2}\right)}{\log _{2}^{1 / 2} 4 / t} \leq 2 \text { for } 0<b \leq 1
$$




$$
\begin{gathered}
\int_{0}^{1} w_{m}(t) \frac{d t}{\psi(t)} \leq \sum_{k=n_{m}}^{n_{m+1}-1} b_{k} \int_{0}^{2^{-k}} \frac{d t}{\psi(t)}=2 \sum_{k=n_{m}}^{n_{m+1}-1} b_{k} \int_{0}^{2^{-k}} \frac{d\left(t^{1 / 2}\right)}{\log _{2}^{1 / 2} 4 / t} \leq \\
\leq 2 \sum_{k=n_{m}}^{n_{m+1}-1} \frac{1}{k+2} \leq 2
\end{gathered}
$$

Therefore (8) and the definition of $F$ imply that $\|1 / \psi\|_{F} \leq 4$, which proves (9).

Next, we put $D_{m}=\left(2^{-n_{m+1}}, 2^{-n_{m}}\right]$ and

$$
v_{m}(t)=w_{m}(t) \chi_{D_{m}}(t)=\sum_{k=n_{m}}^{n_{m+1}-1} b_{k} \chi_{\left(2^{-k-1}, 2^{-k}\right]}(t) \text { for } m=0,1, \ldots
$$

The functions $v_{m}$ are disjoint. Let us show that the norms of $E$ and $F$ are equivalent on their linear hull.

Suppose that

$$
v(t)=\sum_{m=0}^{r} a_{m} v_{m}(t)
$$

Without loss of generality, we can assume that $a_{m} \geq 0$. Consider $w(t)=\max _{0 \leq m \leq r} a_{m} w_{m}(t)$. The function $w(t)$ monotonically decreases on $(0,1]$, and $v(t) \leq w(t)$. Therefore, by (6),

$$
\|v\|_{E} \leq\|w\|_{E} \leq C \max _{0 \leq m \leq r}\left\{a_{m} \max _{n_{m} \leq k<n_{m+1}} b_{k} \psi\left(2^{-k}\right)\right\} .
$$

Since $b_{k} \psi\left(2^{-k}\right)=1$ for $k=0,1,2, .$. , we obtain

$$
\|v\|_{E} \leq C \max _{0 \leq m \leq r} a_{m}
$$

Now, let us estimate $\|v\|_{F}$ from below. By (7), for any $m=0,1, . ., r$ we have

$$
\begin{aligned}
& \int_{0}^{1} v_{m}^{*}(t) w_{m}(t) d t \geq \int_{0}^{1}\left(v_{m}^{*}(t)\right)^{2} d t=\int_{0}^{1} v_{m}^{2}(t) d t= \\
&=\sum_{k=n_{m}}^{n_{m+1}-1} b_{k}^{2} 2^{-k-1}=\frac{1}{2} \sum_{k=n_{m}}^{n_{m+1}-1} \frac{1}{k+2} \geq \frac{1}{4}
\end{aligned}
$$

Hence (8) implies that $\left\|v_{m}\right\|_{F} \geq 1 / 4$. Therefore, by (10),

$$
\|v\|_{F} \geq \max _{0 \leq m \leq r}\left\{a_{m}\left\|v_{m}\right\|_{F}\right\} \geq \frac{1}{4} \max _{0 \leq m \leq r} a_{m} \geq \frac{\|v\|_{E}}{4 C}
$$

This together with (9) means that the norms of the spaces $E$ and $F$ are equivalent on the linear hull of the set of functions $v_{m}(m=0,1, .$.$) . Hence there exists a B>0$ such that, for an arbitrary $a_{m}$,

$$
B^{-1}\left\|\sum_{m=0}^{\infty} a_{m} v_{m}\right\|_{F} \leq\left\|\sum_{m=0}^{\infty} a_{m} v_{m}\right\|_{E} \leq B\left\|\sum_{m=0}^{\infty} a_{m} v_{m}\right\|_{F} .
$$

In other words, the identity inclusion operator $I: E \rightarrow F$ has not the DSS property.

This completes the proof of Theorem 3. 
Remark 3. Theorem 3 shows that relation (A) does not guarantee the presence of a "gap" between spaces sufficient for the corresponding identity inclusion operator to have the DSS property. On the other hand, simple examples show that, even for symmetric spaces with the same fundamental function, this operator may have this property.

For instance, the inclusion of the Lorentz space $\Lambda\left(t^{1 / p}\right)$ into the space $L_{p}$, where $1<p<\infty$, has the DSS property. Indeed, it is easy to show that any sequence of normalized disjoint functions in the Lorentz space contains a subsequence equivalent to the standard basis in $l_{1}$. At the same time, any such sequence in $L_{p}$ is equivalent to the standard basis in $l_{p}$.

In the last section we show that, unlike $(\mathrm{A})$, condition $(\mathrm{B})$ is sufficient for the inclusion operator $I: E \rightarrow F$ of arbitrary symmetric spaces $E$ and $F$ with fundamental functions $\varphi$ and $\psi$, respectively, to be a DSS operator.

\section{$\S 3$. Suffiiency of condition (B) for the operator $I: E \rightarrow F$ to have the DSS property}

Theorem 4. Suppose that functions $\varphi \in G$ and $\psi \in G$ satisfy condition $(A), \delta_{\varphi}<1$, and we have $M(\tilde{\varphi}) \subset \Lambda(\psi)$. Then the operator $I: M(\tilde{\varphi}) \rightarrow \Lambda(\psi)$ has the DSS property.

First, we prove the following auxiliary assertion.

Lemma 1. Under the assumptions of Theorem 4, there exists a function $\rho \in G$ such that

$$
\begin{aligned}
& \text { (1) } \lim _{t \rightarrow 0} \frac{\rho(t)}{\varphi(t)}=0 ; \\
& \text { (2) } M(\tilde{\rho}) \subset \Lambda(\psi) .
\end{aligned}
$$

Proof. Since $\delta_{\varphi}<1$, [8, p.156] implies that

$$
\|x\|_{M(\tilde{\varphi})} \approx \sup _{0<t \leq 1}\left\{\varphi(t) x^{*}(t)\right\} .
$$

Therefore the relation $M(\tilde{\varphi}) \subset \Lambda(\psi)$ is equivalent to

$$
\int_{0}^{1} \frac{d \psi(s)}{\varphi(s)}<\infty
$$

Since the function $\varphi$ is concave, we have

$$
\int_{0}^{1} \frac{d \psi(s)}{\varphi(s)} \approx \sum_{k=0}^{\infty} \frac{\psi\left(2^{-k}\right)-\psi\left(2^{-k-1}\right)}{\varphi\left(2^{-k}\right)}
$$

hence (11) is equivalent to the condition

$$
\sum_{k=0}^{\infty} \frac{\psi\left(2^{-k}\right)-\psi\left(2^{-k-1}\right)}{\varphi\left(2^{-k}\right)}<\infty
$$

Put

$$
a_{k}=\psi\left(2^{-k}\right)-\psi\left(2^{-k-1}\right) \quad \text { and } \quad S_{n}=\sum_{k=n}^{\infty} \frac{a_{k}}{\varphi\left(2^{-k}\right)}
$$


Then $S_{n} \rightarrow$ 0, and [12, Chap. 3, Ex. 12] and (12) imply that

$$
\sum_{k=0}^{\infty} \frac{a_{k}}{\sqrt{S_{k}} \varphi\left(2^{-k}\right)}<\infty
$$

By the definition of upper dilation index, there exist $u>0$ and $C>0$ such that $\delta_{\varphi}+u<1$ and

$$
\mathcal{M}_{\varphi}(t) \leq C t^{\delta_{\varphi}+u / 2}
$$

for all $t \geq 1$. Consider the sequence of numbers

$$
g_{0}=S_{0}, \quad g_{k}=\max \left(S_{k}, 2^{-u} g_{k-1}\right) \text { for } k=1,2, \ldots
$$

Suppose that $g=g(t)$ is a function linear on the intervals $\left[2^{-k-1}, 2^{-k}\right], g\left(2^{-k}\right)=g_{k}$ for $k=0,1, . .$, and $h(t)=\sqrt{g(t)} \varphi(t)$.

Since $\left\{S_{k}\right\}$ decreases, $\left\{g_{k}\right\}$ also decreases; therefore, the functions $g(t)$ and $h(t)$ increase on $(0,1]$. It follows from (15) that, for $j \geq 0$ and $2^{-k-1}<t \leq 2^{-k}$,

$$
\frac{g\left(2^{j} t\right)}{g(t)} \leq \frac{g\left(2^{j-k}\right)}{g\left(2^{-k-1}\right)}=\frac{g_{k-j}}{g_{k+1}}=\frac{g_{k+1-(j+1)}}{g_{k+1}} \leq 2^{(j+1) u} .
$$

Hence, by (14),

$$
\mathcal{M}_{h}\left(2^{j}\right)=\sup _{0<t \leq 2^{-j}} \frac{\varphi\left(2^{j} t\right) \sqrt{g\left(2^{j} t\right)}}{\varphi(t) \sqrt{g(t)}} \leq C 2^{u / 2} 2^{\left(\delta_{\varphi}+u\right) j} \text { for } j=0,1, \ldots
$$

This implies that $\delta_{h}<1$, because $\delta_{\varphi}+u<1$. Therefore, according to [8, p.78], the function $h(t)$ is equivalent to its least concave majorant; we denote this majorant by $\rho(t)$.

The function $\rho$ belongs to $G$, and $\rho\left(2^{-k}\right) \approx h\left(2^{-k}\right)=\sqrt{g_{k}} \varphi\left(2^{-k}\right)$ for $k=0,1,2, .$. ; in addition, since $g_{k} \geq S_{k}$, it follows from (13) that

$$
\sum_{k=0}^{\infty} \frac{a_{k}}{\rho\left(2^{-k}\right)} \leq C_{1} \sum_{k=0}^{\infty} \frac{a_{k}}{\sqrt{S_{k}} \varphi\left(2^{-k}\right)}<\infty
$$

This equivalent to

$$
M(\tilde{\rho}) \subset \Lambda(\psi)
$$

the equivalence is proved in the same way as for the function $\varphi$ (see (11) and (12)).

Finally, relation (15) gives

$$
\lim _{t \rightarrow 0} \frac{\rho(t)}{\varphi(t)} \leq C_{1} \lim _{t \rightarrow 0} \sqrt{g(t)}=\lim _{k \rightarrow \infty} \sqrt{g_{k}}=0 .
$$

This completes the proof of Lemma 1.

Proof of Theorem 4. By Lemma 1, there exists a function $\rho \in G$ such that

$$
M(\tilde{\varphi}) \subset M(\tilde{\rho}) \subset \Lambda(\psi) \text { and } \lim _{t \rightarrow 0} \frac{\rho(t)}{\varphi(t)}=0 .
$$

According to Corollary 2, the operator $I: M(\tilde{\varphi}) \rightarrow M(\tilde{\rho})$ has the DSS property; all the more, it has this property when regarded as an operator from $M(\tilde{\varphi})$ into $\Lambda(\psi)$.

This proves Theorem 4. 
Theorem 4 makes it possible to prove the sufficiency of condition (B) for the identity inclusion operator from an SS $E$ into an $\operatorname{SS} F$ with fundamental functions $\varphi$ and $\psi$, respectively, to have the DSS property.

Theorem 5. Suppose that functions $\varphi \in G$ and $\psi \in G$ satisfy condition (B) and $E$ and $F$ are symmetric spaces with fundamental functions $\varphi$ and $\psi$, respectively. Then $E \subset F$ and $I: E \rightarrow F$ is a DSS operator.

Proof. Let us verify that the assumptions of Theorem 4 hold, i.e., that $\delta_{\varphi}<1$ and that

$$
M(\tilde{\varphi}) \subset \Lambda(\psi) .
$$

First, condition (B) implies the existence of $u>0$ and $C>0$ such that

$$
\frac{\psi(t s) \varphi(s)}{\psi(s) \varphi(t s)} \leq C t^{u}
$$

whenever $0<t \leq 1$ and $0<s \leq 1$. This and the concavity of $\psi$ give

$$
\mathcal{M}_{\varphi}\left(\frac{1}{t}\right) \leq C \mathcal{M}_{\psi}\left(\frac{1}{t}\right) t^{u}=C t^{u-1}
$$

therefore, $\delta_{\varphi} \leq 1-u<1$.

Next, since the function $x^{*}(t)$ decreases, we have

$$
x^{*}(t) \leq \frac{\|x\|_{M(\tilde{\varphi})}}{\varphi(t)} \text { if } x \in M(\tilde{\varphi}) \text { and } 0<t \leq 1 .
$$

Therefore, to prove (16), it suffices to verify that $1 / \varphi \in \Lambda(\psi)$.

By $(17), \psi(t) / \varphi(t) \leq C_{1} t^{u}$. Hence $\psi(t) \rightarrow 0$ as $t \rightarrow 0$, and

$$
\|1 / \varphi\|_{\Lambda(\psi)}=\int_{0}^{1} \frac{\psi^{\prime}(t)}{\varphi(t)} d t \leq \int_{0}^{1} \frac{\psi(t)}{\varphi(t)} \frac{d t}{t} \leq \frac{C_{1}}{u}<\infty .
$$

This proves (16). The above-mentioned extremality of the Lorentz and Marcinkiewicz spaces in the class of symmetric spaces with the same fundamental function [8] implies that $E \subset M(\tilde{\varphi}) \subset \Lambda(\psi) \subset F$. Therefore, $I: E \rightarrow F$ has the DSS property, because it has this property as an operator from $M(\tilde{\varphi})$ into $\Lambda(\psi)$ by Theorem 4 .

This completes the proof of Theorem 5 .

Remark 4. Theorem 5 was proved in [7] for Orlicz spaces under a condition on functions $\varphi$ and $\psi$ somewhat more restrictive than (B), namely, the inequality $\delta_{\varphi}<\gamma_{\psi}$.

The author wishes to express his gratitude to S. Ya. Novikov for useful discussiones. 


\section{References}

[1] A.Pietsch, Operator ideals, Deutscher Verlag der Wissenschaften, Berlin (1980).

[2] A.Grothendieck, Sur certains sous-espaces vectoriels de $L^{p}$, Canad. J. Math., 6, 158-160 (1954).

[3] W.Rudin, Functional analysis, McGraw-Hill, New York (1973).

[4] V.A.Rodin and E.M.Semenov, Rademacher series in symmetric spaces, Anal. Math., 1, No. 3. 207-222 (1975).

[5] F.L.Hernandez and B.Rodrigues-Salinas, On $l^{p}$-complemented copies in Orlicz spaces. 2, Israel J. Math., 68, 27-55 (1989).

[6] A.Zygmund, Trigonometric Series, Vol. 2, Cambridge (1960).

[7] F.L.Hernandez, Disjointly strictly-singular operators, Proc. 19th Winter School on Analysis (Srni). Acta Univ. Carolin. Math. Phys., 31, 35-49 (1990).

[8] S.G.Krein, Yu.I.Petunin and E.M.Semenov, Interpolation of Linear Operators [in Russian], Nauka, Moscow (1978).

[9] M.A.Krasnoselski and Ya.B.Ruticki, Convex functions and Orlicz spaces [in Russian], Fizmatgiz, Moscow (1958).

[10] S.Ya.Novikov, About number index of subspace of symmetric space, in: Studies in the Theory of Functions of Several Real Variables [in Russian], Yaroslav. Gos. Univ., Yaroslavl' (1980), pp. 140-148.

[11] S.Ja.Novikov, Boundary spaces for inclusion map between rearrangement invariant spaces, Proc. 3rd Conf. Function Spaces (Poznan). Collect. Math., 44, 211-215 (1993).

[12] W.Rudin, Principles of Mathematical Analysis, McGraw-Hill, New York (1964). 